\title{
High-frequency impedance of small-angle tapers and collimators
}

\author{
G. Stupakov \\ SLAC National Accelerator Laboratory, Menlo Park, California 94025, USA \\ B. Podobedov \\ Brookhaven National Laboratory, Upton, New York 11973, USA \\ (Received 13 July 2010; published 4 October 2010)
}

\begin{abstract}
Collimators and transitions in accelerator vacuum chambers often include small-angle tapering to lower the wakefields generated by the beam. While the low-frequency impedance is well described by Yokoya's formula (for axisymmetric geometry), much less is known about the behavior of the impedance in the high-frequency limit. In this paper we develop an analytical approach to the high-frequency regime for round collimators and tapers. Our analytical results are compared with computer simulations using the code ECHO.
\end{abstract}

DOI: 10.1103/PhysRevSTAB.13.104401

PACS numbers: 41.60. $-\mathrm{m}$, 03.50.De, 41.20.Jb

\section{INTRODUCTION}

The impedance of small-angle axisymmetric tapers with perfectly conducting walls was first computed analytically by Yokoya in the limit of low frequencies [1]. In this limit the longitudinal impedance is purely imaginary, which means that the beam does not lose energy to radiation. Later works [2,3] generalized Yokoya's approach for rectangular and elliptical cross sections of the transitions. In the opposite limit of very high frequencies, a so-called optical model has been developed [4,5] which predicts a real longitudinal impedance. Simulations show, however, that there is a large range of frequencies between Yokoya's theory and the optical impedance where both theories fail to provide an accurate result. In this paper we address this intermediate regime between the two limiting theories. This paper uses the method developed in an earlier paper by one of the authors [6], which attempted to solve this problem, but failed to take into account the effect of mode transformation in transition regions.

In this paper we consider the geometry of an axisymmetric collimator shown in Fig. 1. It consists of two identical conical tapers of length $l$ connected by a section of a cylindrical waveguide of length $g$. The radius of the pipes outside of the collimator is $b_{1}$, and the radius of the pipe between the tapers is $b_{2}$. We use cylindrical coordinate system $r, z, \phi$ with the origin of the coordinate $z$ situated in the middle of the collimator. The system is then symmetric with respect to reflection in the plane $z=$ 0 [7]. The radius of the collimator $b(z)$ as a function of $z$ is defined by

$$
b(z)= \begin{cases}b_{2}, & 0<|z|<\frac{g}{2}, \\ b_{2}+\left(b_{1}-b_{2}\right) \frac{|z|-g / 2}{l}, & \frac{g}{2}<|z|<l+\frac{g}{2} \\ b_{1}, & |z|>l+\frac{g}{2} .\end{cases}
$$

Throughout this paper we assume that the angle of the collimator $\alpha$ is small,

$$
\alpha=\arctan \frac{b_{1}-b_{2}}{l} \approx \frac{b_{1}-b_{2}}{l} \ll 1 .
$$

We assume that a beam propagates along the axis of the collimator at the speed of light. Our goal is to calculate the longitudinal impedance of the collimator.

\section{THE METHOD}

We will use a method of eigenmodes, in which the electromagnetic radiation field of the beam is represented by a sum of modes of the empty waveguide. It is based on calculation of the energy radiated by the image currents induced by the beam in the walls of the waveguide. In the absence of other losses, the radiated energy is equal to the energy loss of the beam and can be related to the real part of the impedance. The imaginary part of the impedance can then be found using the Kramers-Kronig relations between the imaginary and real parts of the impedance.

The Fourier component of the beam current is (we assume the $e^{-i \omega t}$ time dependence in what follows)

$$
I_{\omega}=I_{0} e^{i k z},
$$

where $\omega$ stands for frequency, $I_{0}$ is the amplitude of the current harmonic, and $k=\omega / c$. Let us denote the timeaveraged intensity of radiation of this current from the collimator region by $P_{\omega}$. The real part of the impedance is then given by the following relation (see, e.g., [8]):

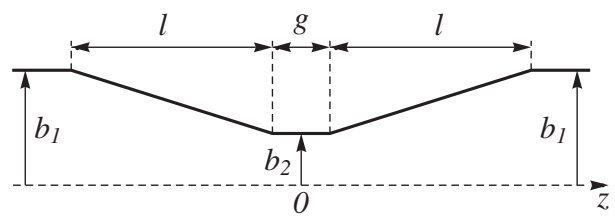

FIG. 1. Geometry of an axisymmetric collimator. 


$$
\operatorname{Re} Z(\omega)=\frac{2 P_{\omega}}{I_{0}^{2}} .
$$

The radiation is due to the image currents induced in the perfectly conducting walls in the taper regions where the walls are not parallel to the $z$ axis. It is convenient to represent the total electric field of the beam current (2) inside the taper as a sum of the vacuum field, $\boldsymbol{E}^{\mathrm{vac}}$, and the radiation field $\boldsymbol{E}^{\mathrm{rad}}, \boldsymbol{E}=\boldsymbol{E}^{\mathrm{vac}}+\boldsymbol{E}^{\mathrm{rad}}$, where for an on-axis beam

$$
\boldsymbol{E}^{\mathrm{vac}}=\hat{\boldsymbol{r}} \frac{2 I_{0}}{r c} e^{i k z},
$$

with $\hat{\boldsymbol{r}}$ being a unit vector in the radial direction of the cylindrical coordinate system.

The radiation field $\boldsymbol{E}^{\text {rad }}$ satisfies Maxwell's equation with the boundary condition that requires the tangential component of the total electric field on the wall to vanish

$$
\left.\boldsymbol{E}_{t}^{\mathrm{rad}}\right|_{\text {wall }}=-\left.\boldsymbol{E}_{t}^{\mathrm{vac}}\right|_{\text {wall }} \text {. }
$$

It is convenient to replace the boundary conditions (5) for a nonvanishing tangential component of the radiation electric field on the wall by the surface magnetic current $\boldsymbol{i}^{\mathrm{mag}}$ located inside the waveguide infinitesimally close to the wall $[9,10]$. The magnitude and direction of the magnetic current is given by

$$
\boldsymbol{i}^{\mathrm{mag}}=\frac{c}{4 \pi} \boldsymbol{n} \times\left.\boldsymbol{E}_{t}^{\mathrm{rad}}\right|_{\text {wall }}=-\frac{c}{4 \pi} \boldsymbol{n} \times\left.\boldsymbol{E}_{t}^{\mathrm{vac}}\right|_{\text {wall }},
$$

where $\boldsymbol{n}$ is the unit vector normal to the surface and directed towards the metal. Note that the magnetic current exists only inside the tapers and vanishes in the region where the wall is parallel to the $z$ axis.

Inside the waveguide, the radiation field excited by the magnetic currents can be represented as a sum of eigenmodes,

$$
\boldsymbol{E}^{\mathrm{rad}}=\sum_{n} a_{n} \boldsymbol{E}_{n}^{+},
$$

where $a_{n}$ is the amplitude and $\boldsymbol{E}_{n}^{+}$is the electric field of the $n$th eigenmode, propagating in the positive direction of the $z$ axis. A similar expansion in terms of the amplitudes $a_{n}$ is also valid for the magnetic field. Note that, in general, the sum in (7) also includes modes $\boldsymbol{E}_{n}^{-}$propagating in the backward direction [9]. However, in the limit of high frequency, the modes that make a dominant contribution to the impedance propagate in the forward direction, and the backward propagating modes can be neglected.

The norm $N_{n}$ of the mode $n$ is defined as

$$
N_{n}=\frac{c}{4 \pi} \int \boldsymbol{m} \cdot\left(\boldsymbol{E}_{n}^{+} \times \boldsymbol{H}_{n}^{-}-\boldsymbol{E}_{n}^{-} \times \boldsymbol{H}_{n}^{+}\right) d S,
$$

where the integral is taken over the cross section of the waveguide and the unit vector $\boldsymbol{m}$ is perpendicular to the integration surface and points in the direction of propagation. It can be shown that $N_{n}$ does not depend on the choice of position of the cross section. The right-hand side of Eq. (8) can be considered as a scalar product of the two fields $\boldsymbol{E}_{n}^{+}, \boldsymbol{H}_{n}^{+}$and $\boldsymbol{E}_{n}^{-}, \boldsymbol{H}_{n}^{-}$. One can show [9] that the scalar product of two different modes is equal to zero, so that a generalization of Eq. (8) for $n \neq j$ is

$$
\frac{c}{4 \pi} \int \boldsymbol{m} \cdot\left(\boldsymbol{E}_{n}^{+} \times \boldsymbol{H}_{j}^{-}-\boldsymbol{E}_{j}^{-} \times \boldsymbol{H}_{n}^{+}\right) d S=N_{n} \delta_{n j} .
$$

We will use this equation in the next section.

The energy radiated by the current $P_{\omega}$ can be written as a sum over all possible modes,

$$
P_{\omega}=\sum_{n} P_{n}\left|a_{n}\right|^{2},
$$

where $P_{n}$ is the energy flow in the mode of unit amplitude.

\section{EIGENMODES IN THE COLLIMATOR}

As described in the previous section, to calculate the excitation of electromagnetic field by the beam, one needs to know the eigenmodes of the complete circuit. Analytical expressions for eigenmodes are available for cylindrical and conical waveguides; however, there is no a compact expression for eigenmodes of a collimator shown in Fig. 1. More precisely, a single conical mode that propagates in the left taper of the collimator experiences transformation at the transition to the straight central section, generating several modes in the central part. Each of these modes, in turn, experiences a transformation at the second transition from the cylindrical waveguide to the right taper, resulting in multiple conical modes in the right taper. We will have to take these transformation processes into account in our analysis.

For calculation of the longitudinal impedance, one only needs axisymmetric TM modes. In the cylindrical central part of the collimator, the modes propagating in the positive direction, $\boldsymbol{E}_{n}^{+}, \boldsymbol{H}_{n}^{+}$, are given by the following equations:

$$
\begin{aligned}
E_{z, n}^{+} & =\frac{j_{n}^{2}}{b_{2}^{2}} J_{0}\left(j_{n} \frac{r}{b_{2}}\right) e^{i \phi_{n}(z)} \\
E_{r, n}^{+} & =-\frac{i j_{n} k_{n}}{b_{2}} J_{1}\left(j_{n} \frac{r}{b_{2}}\right) e^{i \phi_{n}(z)} \\
H_{\phi, n}^{+} & =-\frac{i \omega j_{n}}{b_{2} c} J_{1}\left(j_{n} \frac{r}{b_{2}}\right) e^{i \phi_{n}(z)},
\end{aligned}
$$

where $n$ is the mode index, $n=1,2, \ldots, J_{0}$, and $J_{1}$ are the Bessel functions, $j_{n}$ is the $n$th root of $J_{0}$, and $k_{n}=$ $\left(\omega^{2} / c^{2}-j_{n}^{2} / b_{2}^{2}\right)^{1 / 2}$. The phase of the mode is equal to $\phi_{n}(z)=k_{n} z$. The modes propagating in the negative direction, $\boldsymbol{E}_{n}^{-}, \boldsymbol{H}_{n}^{-}$, are obtained from the forward modes by changing the signs of $\phi_{n}$ and $E_{r, n}^{+}$in (11).

A simple calculation gives the norm (8) of the mode $n$ :

$$
N_{n}=\frac{1}{2} \omega k_{n} j_{n}^{2} J_{1}^{2}\left(j_{n}\right),
$$

with the energy flow in the mode equal to $P_{n}=N_{n} / 4$. 
In the limit of high frequency, it turns out that only the modes that propagate at small angles to the axis of the system make the dominant contribution to the impedance (so-called paraxial approximation, see [11]). In this approximation one can neglect the transverse component $j_{n} / b_{2}$ of the wave vector and replace $k_{n}$ by $k$ everywhere in Eqs. (11) and (12), except for the phase $\phi_{n}$.

Analytical expressions for eigenmodes of the electromagnetic field are also available for conical geometry (see, e.g., [9]). In the general case of arbitrary cone angle $\alpha$ and arbitrary frequency $\omega$, they involve the Legendre and Bessel functions. We will use here a simplified version of these functions valid in the limit of a small angle $\alpha$ and high frequency $\omega$, replacing $k_{n}$ by $k$ where possible [see explanation after Eq. (12)]. The derivation of these modes is given in the Appendix. In this limit, the conical eigenmodes (which we mark by the tilde below) are similar to the cylindrical ones, and, in our cylindrical coordinate system, they can be written as follows:

$$
\begin{aligned}
\tilde{E}_{z, n}^{+} & =\frac{j_{n}^{2}}{b^{2}} J_{0}\left(j_{n} \frac{r}{b}\right) e^{i \phi_{n}(z)+i k r^{2} / 2 R(z)} \\
\tilde{E}_{r, n}^{+} & =-\frac{i j_{n} k}{b} J_{1}\left(j_{n} \frac{r}{b}\right) e^{i \phi_{n}(z)+i k r^{2} / 2 R(z)} \\
\tilde{H}_{\phi, n}^{+} & =-\frac{i \omega j_{n}}{b c} J_{1}\left(j_{n} \frac{r}{b}\right) e^{i \phi_{n}(z)+i k r^{2} / 2 R(z)},
\end{aligned}
$$

where the phase $\phi_{n}$ is now determined from the differential equation $d \phi_{n} / d z=\left[\omega^{2} / c^{2}-j_{n}^{2} / b(z)^{2}\right]^{1 / 2}$. The factor $R(z)$ in the above equations is the curvature radius of the spherical wave fronts of the modes in the conical regions; it is equal $R(z)=\left[\arctan b^{\prime}(z) / b(z)\right]^{-1} \approx b(z) / b^{\prime}(z)$. Note that, due to the linear dependence of $b(z)$ in the tapers, $b^{\prime}=$ const. The sign of $R$ is important: it is negative in the left taper, corresponding to converging wave fronts of the modes, and is positive in the right taper, where the wave fronts are diverging from the center of the collimator. Because Eqs. (13) differ from Eqs. (11) only by a phase factor, the norm for the conical modes is the same as for the cylindrical ones, given by (12). The relation $P_{n}=N_{n} / 4$ holds as well.

For the phase $\phi_{n}(z)$ in (13) we have

$$
\phi_{n}(z)=\int_{0}^{z}\left[\frac{\omega^{2}}{c^{2}}-\frac{j_{n}^{2}}{b\left(z^{\prime}\right)^{2}}\right]^{1 / 2} d z^{\prime}
$$

The conical modes propagating in the negative direction are obtained from (13) by changing the signs of $\phi_{n}$ and $E_{r, n}^{+}$.

The small angle of the collimator, as was pointed out in [6], allows one to neglect the reflection of eigenmodes at the transitions between the cylindrical and conical regions. However, it does not preclude mode transformation at these transitions, and we will account for this below.

\section{AMPLITUDES OF THE MODES AND MODE TRANSFORMATION}

Calculation of the mode amplitudes in the collimator is performed in several steps. At $z=-(l+g / 2)$ where the beam enters the collimator, there is no radiation field present, hence $a_{n}=0$ in Eq. (7).

In the left taper, where there are magnetic currents (6), the amplitudes $a_{n}$ depend on $z$. The values of the amplitudes at the exit from the left taper, $z=-g / 2-\epsilon$ (an infinitesimally small $\epsilon$ here indicates a location right before the exit from the taper), are given by the following integrals [9]:

$$
a_{n}^{(1)}=-\frac{1}{N_{n}} \int_{\mathrm{left}} i^{\mathrm{mag}} \cdot \tilde{\boldsymbol{H}}_{n}^{-} d S,
$$

where $\boldsymbol{H}_{n}^{-}$is the magnetic field of the $n$th eigenmode propagating in the negative direction, $d S$ is an infinitesimal element of the surface area, and the integration covers the wall area in the left taper where the magnetic current resides.

At the transition point $z=-g / 2$, the modes will be transformed from conical to cylindrical ones, and the amplitudes of the modes will be linearly transformed from $a_{n}^{(1)}$ at $z=-g / 2-\epsilon$ to $a_{n}^{(2)}$ at $z=-g / 2+\epsilon$,

$$
a_{n}^{(2)}=\sum_{j} S_{n j} a_{j}^{(1)}
$$

We will discuss below how the matrix elements $S_{n j}$ are computed.

The next mode transformation occurs at the transition $z=g / 2$ with the new amplitudes $a_{n}^{(3)}$,

$$
a_{n}^{(3)}=\sum_{j} R_{n j} a_{j}^{(2)} .
$$

Finally, radiation of the magnetic currents in the right taper will add to $a_{n}^{(3)}$ :

$$
a_{n}^{(4)}=a_{n}^{(3)}-\frac{1}{N_{n}} \int_{\text {right }} i^{\mathrm{mag}} \cdot \tilde{\boldsymbol{H}}_{n}^{-} d S .
$$

The amplitudes $a_{n}^{(4)}$ are the final values that should be used in Eq. (10) to calculate the radiated power.

We now use Eqs. (4), (6), (12), and (13), to compute $a_{n}^{(1)}$ in (15)

$$
a_{n}^{(1)}=-\frac{2 i I_{0} \alpha}{c k j_{n} J_{1}\left(j_{n}\right)} \int_{-l-g / 2}^{-g / 2} \frac{d z}{b(z)} e^{i k z-i \phi_{n}(z)+i k b(z) \alpha / 2},
$$

where we have used the small-angle approximation, $\alpha \ll$ 1 , and $b^{\prime} \approx-\alpha$ in the left taper. Taking into account the symmetry of the collimator and performing a similar cal- 
culation for the second term on the right-hand side of Eq. (18), one finds that it is equal to the complex conjugate of (19).

To find the matrix elements $S_{n j}$ in Eq. (16), we note that due to the continuity of the field at $z=-g / 2$ it can be expanded into the conical eigenmodes as well as the cylindrical modes:

$$
\sum_{n} a_{n}^{(2)} \tilde{\boldsymbol{E}}_{n}^{+}=\sum_{j} a_{j}^{(1)} \boldsymbol{E}_{j}^{+}
$$

with a similar continuity equation holding for the magnetic fields. Using the orthogonality property of the modes with respect to the scalar product (9), we find

$$
a_{n}^{(2)}=\frac{c}{4 \pi N_{n}} \sum_{j} a_{j}^{(1)} \int \boldsymbol{m} \cdot\left(\boldsymbol{E}_{j}^{+} \times \tilde{\boldsymbol{H}}_{n}^{-}-\tilde{\boldsymbol{E}}_{n}^{-} \times \boldsymbol{H}_{j}^{+}\right) d S,
$$

which defines the matrix elements in Eq. (16). The matrix elements $R_{n j}$ in Eq. (17) could be found in a similar fashion.

\section{NUMERICAL RESULTS}

The impedance calculation algorithm described above was implemented in MATHEMATICA [12]. In numerical calculations, the infinite sums involved in Eqs. (10), (16), and (17) are truncated, and only first $N_{m} \approx 10$ lowest modes are used to calculate the impedance. By varying $N_{m}$ we verified that the result does not depend on the exact value of $N_{m}$.

To illustrate our method, we have chosen the following collimator geometry: $l=g=3 \mathrm{~cm}, b_{1}=2 b_{2}=0.5 \mathrm{~cm}$, so that the collimator angle is $\alpha=4.7$ degrees. The real part of the impedance computed from the beam pipe cutoff, $f_{c}=j_{1} c / 2 \pi b_{2}=46 \mathrm{GH} z$, up to the frequency $f_{\max }=$ $3.9 \mathrm{THz}$ is shown in Fig. 2 in solid blue. At higher frequencies this impedance approaches the optical model value, $Z_{\mathrm{opt}}=\left(Z_{0} / \pi\right) \log \left(b_{1} / b_{2}\right)=83 \Omega$.

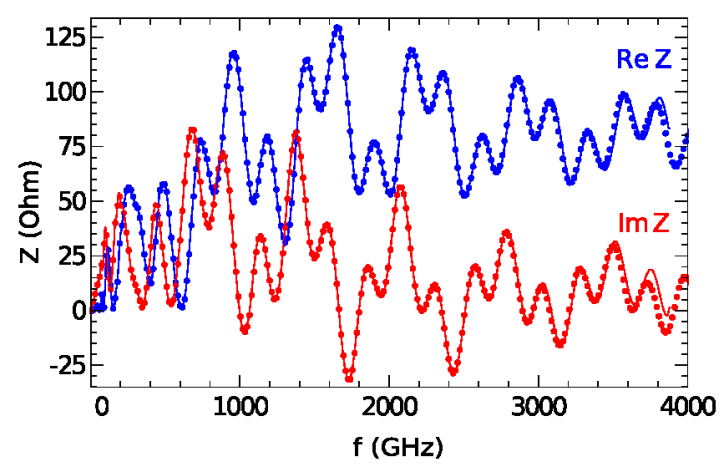

FIG. 2. Collimator impedance: present theory is shown with solid line, the result of simulations with ECHO are shown with dots.

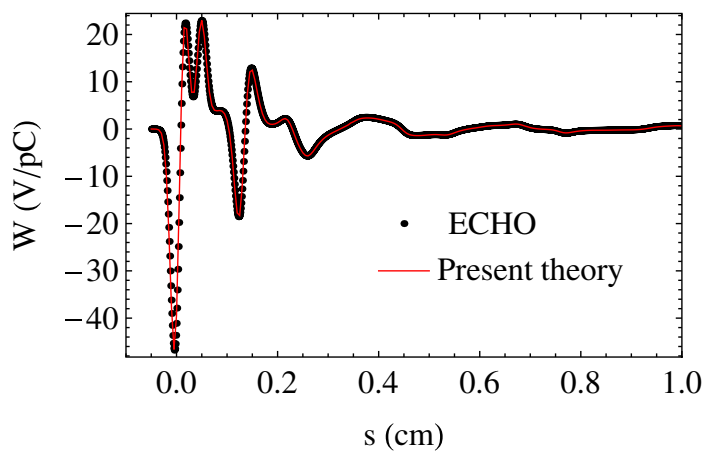

FIG. 3. Wake potential of a $\sigma_{z}=100 \mu \mathrm{m}$ bunch.

While our algorithm directly finds only the real part of impedance, we can find the imaginary part by making use of causality, which relates imaginary and real parts of the impedance via the Hilbert transforms (Kramers-Kronig relations) [8]. To proceed, we need to define $\operatorname{Re} Z$ for all frequencies, so we set it to zero below $f_{c}$, and set it equal to the optical model value above $f_{\max }$. $\operatorname{Im} Z$ calculated by the Hilbert transform of this $\operatorname{Re} Z$ is shown in Fig. 2 in solid red. Below the cutoff frequency it ends up very close to the Yokoya value. For comparison, we plot impedances calculated from a Fourier-transformed wake potential of a $\sigma_{z}=$ $20 \mu \mathrm{m}$ Gaussian bunch computed by the EM code ECHO based on a finite integration technique [13]. Running ECHO we usually used the grid size from 5 to 200 times smaller than $\sigma_{z}$ and made sure that the numerical result converged and did not change with further refining of the mesh. One can see a very good agreement between our approach and the ECHO results.

Since our algorithm allows one to accurately find the impedance over a very broad frequency range, we can use inverse Fourier transform to reconstruct the wake potential of a short bunch. For instance, for a Gaussian bunch with rms length $\sigma_{z}=100 \mu \mathrm{m}$, we obtain the wake potential shown in Fig. 3, plotted with the ECHO result for comparison. Again, we observe a perfect agreement between the two.

Finally, in Fig. 4 we present the loss factor and the maximum absolute value of the wake potential as a func-

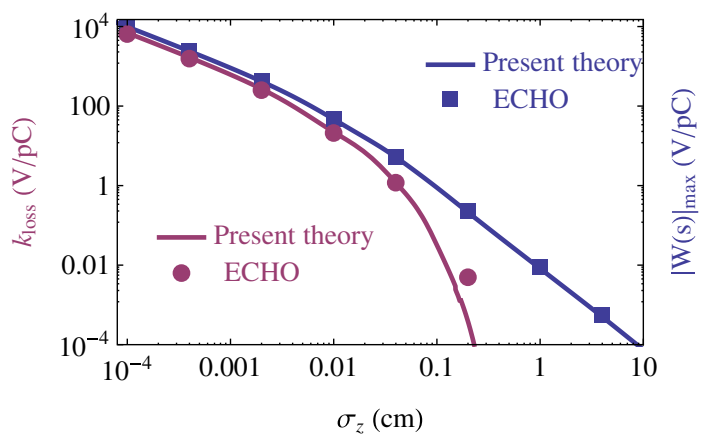

FIG. 4. Loss factor and maximum of the wake potential. 
tion of bunch length. As we expect from the optical model, for short bunches, both quantities scale as $\sigma_{z}^{-1}$, while in the opposite, Yokoya regime, $|W(s)|_{\max } \propto \sigma_{z}^{-2}$ and the loss becomes exponentially small. In the intermediate region [roughly 2 magnitude orders in $\sigma_{z}$ with corresponding changes of 3 or more orders in magnitude in $|W(s)|_{\max }$ and $k_{\text {loss }}$ the scaling is more complex, and, to our knowledge, it is not described by any existing analytical treatments. Our new approach comfortably fills this gap.

\section{CONCLUSIONS}

In conclusion, we developed a novel analytical approach to find the impedance of (small-angle) tapered collimators in axially symmetric geometry. Impedance can be found over a very broad frequency range, from DC to highfrequency optical model limit, thus allowing one to reconstruct the wake potential of short bunches. We note that this algorithm is also applicable to convex (cavitylike) structures. Our general method can also be used for tapers with large angle $\alpha$; however, this would require working with exact eigenfunctions in the conical region, given by Eq. (A1), instead of simpler expressions, Eq. (13).

We plan to apply our method to 3D geometries in the future.

\section{ACKNOWLEDGMENTS}

This work was supported by the U.S. Department of Energy under Contracts No. DE-AC02-76SF00515 and No. DE-AC02-98CH10886.

\section{APPENDIX: DERIVATION OF APPROXIMATE EIGENMODES FOR A CONICAL WAVEGUIDE}

Consider a conical waveguide with its associated spherical coordinate system $\rho, \theta$, and $\phi$, such that the origin of the system is located at the vertex of the cone, see Fig. 5. The metal surface of the waveguide is located at $\theta=\alpha$, and the electromagnetic fields occupy the region $\theta<\alpha$.

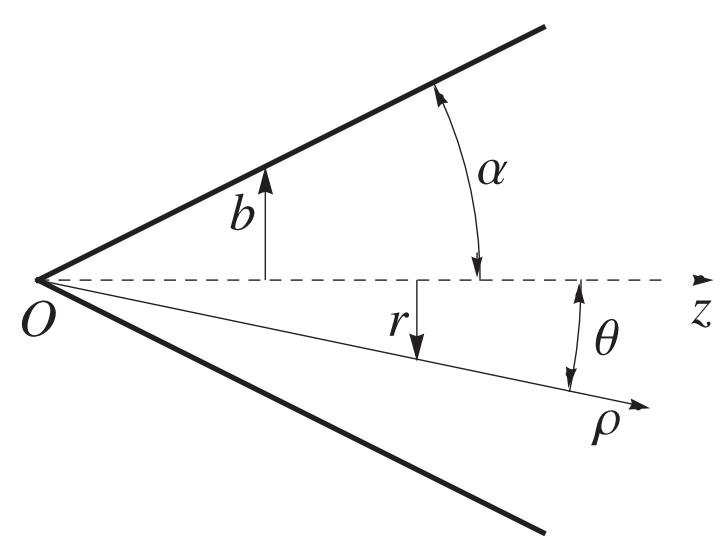

FIG. 5. Spherical and cylindrical coordinate systems associated with a conical waveguide.
The transformation from the spherical coordinate system to the cylindrical one $r, \phi, z$ employed in the main body of our paper is given by $z=\rho \cos \theta$ and $r=\rho \sin \theta$, where $z$ is now measured from the vertex of the cone.

We are interested in axisymmetric (no $\phi$ dependence) TM eigenmodes that can propagate in the waveguide. These modes have three components of the field: $E_{\rho}, E_{\theta}$, and $H_{\phi}$. The modes propagating away from the vertex in the positive direction of $\rho$ (denoted by the superscript "+") are given by the following equations [9]:

$$
\begin{gathered}
E_{\rho, \nu}^{+}=\frac{\nu(\nu+1)}{\rho^{2}} U_{\nu}^{+}(\rho, \theta), \quad E_{\theta, \nu}^{+}=\frac{1}{\rho} \frac{\partial^{2} U_{\nu}^{+}}{\partial \rho \partial \theta}, \\
H_{\phi, \nu}^{+}=\frac{i k}{\rho} \frac{\partial U_{\nu}^{+}}{\partial \theta},
\end{gathered}
$$

where

$$
U_{\nu}^{+}(\rho, \theta)=\sqrt{\frac{\pi k \rho}{2}} H_{\nu+(1 / 2)}^{(1)}(k \rho) P_{\nu}[\cos (\theta)],
$$

with $H_{\nu+(1 / 2)}^{(1)}$ the Hankel function of the first kind of order $\nu+\frac{1}{2}$. The eigenvalues $\nu$ are found from the requirement of the zero tangential electric field at the metal surface, $P_{\nu}[\cos (\alpha)]=0$.

The eigenmodes propagating in the negative direction are given by (A1) with $U_{\nu}^{+}$replaced by $U_{\nu}^{-}$. The function $U_{\nu}^{-}$is given by (A2) in which $H_{\nu+(1 / 2)}^{(1)}$ is replaced by the Hankel function of the second kind $H_{\nu+(1 / 2)}^{(2)}$.

In the limit of small angles $\alpha$ the eigenvalues $\nu$ are large, and we can use the approximation $P_{\nu}[\cos (\theta)] \approx J_{0}[(\nu+$ $\left.\frac{1}{2}\right) \theta$ ], where $J_{0}$ is the Bessel function of zero order, and approximate $\nu(\nu+1) \approx\left(\nu+\frac{1}{2}\right)^{2}$. We then can write

$$
\begin{aligned}
E_{\rho, \nu}^{+} & =\frac{\mu^{2}}{\rho^{2}} J_{0}(\mu \theta) \sqrt{\frac{\pi}{2} x} H_{\mu}^{(1)}(x) \\
E_{\theta, \nu}^{+} & =-\frac{\mu k}{\rho} J_{1}(\mu \theta)\left(\sqrt{\frac{\pi}{2}} x H_{\mu}^{(1)}(x)\right)^{\prime} \\
H_{\phi, \nu}^{+} & =-\frac{i \mu k}{\rho} J_{1}(\mu \theta) \sqrt{\frac{\pi}{2}} x H_{\mu}^{(1)}(x),
\end{aligned}
$$

where $x=k \rho$ and $\mu=\nu+\frac{1}{2}$ and the prime denotes the derivative with respect to $x$. The boundary condition now takes the form $J_{0}(\mu \alpha)=0$, from which it follows that

$$
\mu=\frac{j_{n}}{\alpha},
$$

where $j_{n}$ is the $n$th root of $J_{0}$. From (A4) we see that indeed small $\alpha$ means $\mu \gg 1$.

For large values of $x$, one can use the asymptotic expression [14]

$$
H_{\mu}^{(1)}(x) \approx \sqrt{\frac{2}{\pi x}} e^{i \psi(x)},
$$


with

$$
\psi(x) \approx x-\frac{\pi}{2} \mu-\frac{\pi}{4}+\frac{4 \mu^{2}-1}{8 x} .
$$

Substituting (A4) and (A5) into (A3) we obtain

$$
\begin{aligned}
E_{\rho, \nu}^{+} & =\frac{j_{n}^{2}}{b^{2}} J_{0}\left(j_{n} \frac{r}{b}\right) e^{i \psi(x)} \\
E_{\theta, \nu}^{+} & =-\frac{i j_{n} k}{b} J_{1}\left(j_{n} \frac{r}{b}\right) e^{i \psi(x)} \\
H_{\phi, \nu}^{+} & =-\frac{i j_{n} k}{b} J_{1}\left(j_{n} \frac{r}{b}\right) e^{i \psi(x)},
\end{aligned}
$$

where in the derivative with respect to $x$ we used an approximation $\psi^{\prime} \approx 1$ and replaced $b=\alpha \rho$ and $r / b=$ $\theta / \alpha$ (see Fig. 5), where $b$ is the radius of the metal surface in the cylindrical coordinate system. The last two equations are approximations to the exact equalities $b=\rho \sin \alpha$ and $r / b=\sin \theta / \sin \alpha$ in the limit $\theta, \alpha \ll 1$.

To prove that Eqs. (A7) are identical to (13), we need to show that the phase $\psi$ given by (A6) can be also represented as

$$
\psi=\phi_{n}(z)+\frac{k r^{2}}{2 R}
$$

with

$$
\frac{d \phi_{n}}{d z}=\left[k^{2}-\frac{j_{n}^{2}}{b(z)^{2}}\right]^{1 / 2} \approx k-\frac{j_{n}^{2}}{2 k b(z)^{2}} .
$$

Observing that $x=k \rho=k z / \cos \theta \approx k z\left(1+\theta^{2} / 2\right) \approx$ $k z+k r^{2} / 2 z$ we substitute it to (A6) and neglect 1 in comparison with $4 \mu^{2}$ in the last term

$$
\begin{aligned}
\psi(x) & \approx k z+\frac{k r^{2}}{2 z}+\frac{\mu^{2}}{2 x}+\text { const } \\
& \approx k z+\frac{k r^{2}}{2 z}+\frac{j_{n}^{2}}{2 k \alpha^{2} \rho}+\text { const } \\
& \approx k z+\frac{k r^{2}}{2 z}+\frac{j_{n}^{2}}{2 k \alpha b}+\text { const. }
\end{aligned}
$$

The second term in these equations is associated with the quantity $k r^{2} / 2 R$ in (A8) because the coordinate $z$ measured from the vertex is equal to the curvature of the wave fronts defined in (13) as $R$. The sum of the first and the third term indeed satisfies Eq. (A9) if one takes into account $d b(z) / d z=\alpha$.

[1] K. Yokoya, CERN Technical Report No. SL/90-88, 1990.

[2] B. Podobedov and S. Krinsky, Phys. Rev. ST Accel. Beams 10, 074402 (2007).

[3] G. Stupakov, Phys. Rev. ST Accel. Beams 10, 094401 (2007).

[4] G. Stupakov, K. Bane, and I. Zagorodnov, Phys. Rev. ST Accel. Beams 10, 054401 (2007).

[5] K. Bane, G. Stupakov, and I. Zagorodnov, Phys. Rev. ST Accel. Beams 10, 074401 (2007).

[6] G. Stupakov, SLAC Report No. SLAC-PUB-7039, 1995.

[7] Reflection symmetry is not required for our method to work, but it is used in this paper for simplicity.

[8] A. W. Chao, Physics of Collective Beam Instabilities in High Energy Accelerators (Wiley, New York, 1993).

[9] L. A. Vainshtein, Electromagnetic Waves (Radio i svyaz', Moscow, 1988), in Russian.

[10] R. E. Collin, Field Theory of Guided Waves (IEEE, New York, 1991), 2nd ed.

[11] G. Stupakov, New J. Phys. 8, 280 (2006).

[12] MATHEMATICA version 7.0, Wolfram Research, Inc., 2008.

[13] I. Zagorodnov and T. Weiland, Phys. Rev. ST Accel. Beams 8, 042001 (2005).

[14] I. Gradshteyn and I. Ryzhik, Table of integrals, series, and products (Academic Press, New York, 2000), 6th ed. 